\title{
Tratamento cirúrgico da rotura de parede livre do ventrículo esquerdo após infarto agudo do miocárdio
}

\author{
Carlos A. C. ABREU FILHO*, Luís Alberto O. DALLAN*, Luiz Augusto F. LISBOA*, \\ Fernando PLATANIA**, José Carlos. R. IGLÉZIAS*, Richard H. CABRAL*, Rogério BORDALLO*, \\ Luís Augusto P. DALLAN*, Sérgio Almeida de OLIVEIRA*
}

RBCCV 44205-566

\begin{abstract}
Abreu Filho C A C, Dallan L A O, Lisboa L A F, Platania F, Iglézias J C R, Cabral R H, Bordallo $R$, Dallan L A P, Oliveira S A - Tratamento cirúrgico da rotura de parede livre do ventrículo esquerdo após infarto agudo do miocárdio. Rev Bras Cir Cardiovasc 2002; 17(1): 6-12.

RESUMO: Casuística e Metodos: Entre janeiro de 1983 e maio de 1999, 12.405 pacientes com diagnóstico de infarto agudo do miocárdio (IAM) foram atendidos no InCor. Destes, $127(1,02 \%)$ apresentaram rotura de parede livre do ventrículo esquerdo como complicação do IAM. A rotura miocárdica aguda ocorreu em $98(77,1 \%)$ pacientes e a subaguda em $29(22,9 \%)$.

Resultados: Foram operados 5 pacientes na forma aguda, com $80 \%$ de mortalidade e 19 na forma subaguda, com $15,8 \%$ de mortalidade. A sobrevida pós-operatória abrangendo os dois grupos foi de $70,8 \%$.

Conclusão: Concluímos que a rotura de parede livre do ventrículo esquerdo é uma grave complicação do IAM, necessitando de atuação imediata. Nas formas agudas, observa-se rápida deterioração hemodinâmica do paciente, muitas vezes sem tempo hábil para tentativa de correção cirúrgica. As formas subagudas podem ser detectadas e monitorizadas através de exames ecocardiográficos seriados após o IAM. Nestes casos a intervenção cirúrgica precoce, muitas vezes sem a necessidade de instalação de circulação extracorpórea, tem contribuído para a sobrevida da maioria dos pacientes.
\end{abstract}

DESCRITORES: Ventrículo cardíaco, cirurgia. Infarto do miocárdio, complicações. Rotura cardíaca pós infarto.

\section{INTRODUÇÃO}

A rotura cardíaca foi descrita por William Harvey, em $1647^{(1)}$. Em 1850, Joseph Hodgron estabeleceu a relação entre a rotura cardíaca e a coronariopatia obstrutiva. Com alta mortalidade, a história natural dessa doença começou a mudar a partir de 1972, com os primeiros relatos de correções cirúrgicas com sucesso (2).

A rotura cardíaca é uma grave complicação do
IAM, responsável por 16 a $21 \%$ dos óbitos em unidades coronarianas ${ }^{(3)}$, sendo a segunda causa mais freqüente de morte após o IAM, superada apenas pelo choque cardiogênico ${ }^{(2-4)}$.

Sua forma aguda (Figura 1) é caracterizada pela rotura súbita e hemorragia maciça para a cavidade pericárdica, seguida de morte na maioria dos casos. Em alguns pacientes, entretanto, a hemorragia para o pericárdio pode ser lenta e repetitiva, com formação de trombos entre o epicárdio e a cavidade

\footnotetext{
Trabalho realizado no Instituto do Coração do Hospital das Clínicas da Faculdade de Medicina da Universidade de São Paulo. São Paulo, SP, Brasil. Recebido para publicação em maio de 2001.

*Do Instituto do Coração do Hospital das Clínicas da Faculdade de Medicina da Universidade de São Paulo. São Paulo, SP.

** Do Hospital Ana Costa. Santos, SP.

Endereço para correspondência: Carlos Abreu Filho. Av. Dr. Enéas de Carvalho Aguiar, 44 - Cerqueira César, São Paulo, SP, Brasil - CEP 05403 - 000 Tel. (11) 3069-5014. e-mail: dcimalu@incor.usp.br
} 
Abreu Filho C A C, Dallan L A O, Lisboa L A F, Platania F, Iglézias J C R, Cabral R H, Bordallo R, Dallan L A P, Oliveira S A Tratamento cirúrgico da rotura de parede livre do ventrículo esquerdo após infarto agudo do miocárdio. Rev Bras Cir Cardiovasc 2002; 17(1): 6-12.

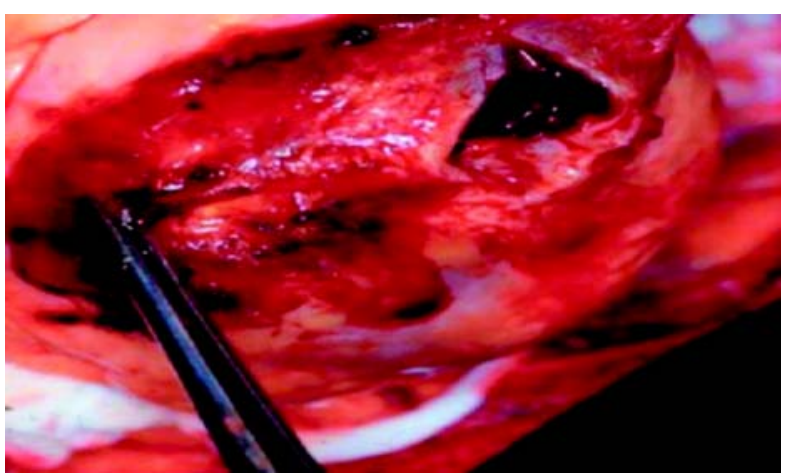

Fig. 1 - Rotura cardíaca aguda.

pericárdica, que contém a hemorragia. Nessas situações, a rotura cardíaca é denominada subaguda (Figura 2), e o paciente pode sobreviver por horas ou dias, o que abre a possibilidade para eventual intervenção cirúrgica com sucesso.

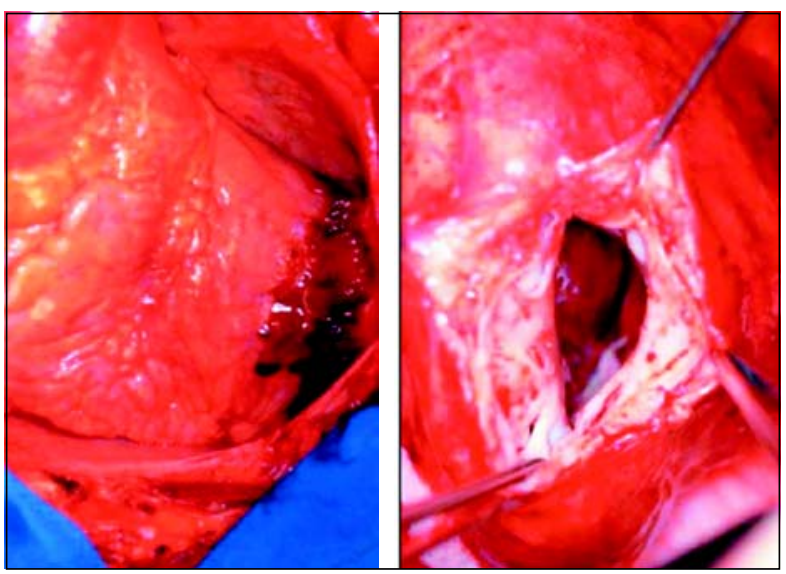

Fig. 2 - Rotura cardíaca sub-aguda.

Em casos especiais, a organização dos trombos pode ocupar um determinado espaço no interior do pericárdio, em contato direto com a cavidade ventricular esquerda, originando o chamado pseudoaneurisma ventricular.

O desenvolvimento e o uso rotineiro dos métodos de diagnóstico por imagem, em especial do ecocardiograma, tem aumentado a possibilidade de diagnósticos precoces, possibilitando seu tratamento cirúrgico com sucesso (2).

O objetivo deste trabalho é avaliar os resultados imediatos do tratamento cirúrgico da rotura de parede livre ventricular após o IAM nas formas aguda e subaguda.

\section{CASUÍSTICA E MÉTODOS}

Entre janeiro de 1983 e maio de 1999, foram atendidos no InCor 12.405 pacientes com diagnóstico de IAM. Destes, $127(1,02 \%)$ apresentaram rotura de parede livre do ventrículo esquerdo como complicação do infarto agudo do miocárdico. A faixa etária média foi de 68,4 anos, variando de 35 a 85 anos. Pudemos observar o predomínio da raça branca $(91,3 \%)$ e do sexo feminino $(52,2 \%)$.

A maioria dos pacientes já adentrou a unidade de emergência em situação crítica ou mesmo em parada cardiorrespiratória, o que impossibilitou sua investigação mais criteriosa. $O$ exame ecocardiográfico transtorácico foi realizado sempre que possível, na tentativa de se estabelecer o diagnóstico, assim como de se analisar a função contrátil das câmaras cardíacas.

O diagnóstico clínico foi estabelecido no préoperatório em 27 (21,3\%) pacientes. Em 6 (4,7\%) pacientes a rotura cardíaca foi constatada no intraoperatório e, nos demais 94 (74\%), foi detectada ou confirmada apenas nos estudos de necropsia.

Vinte e quatro $(18,9 \%)$ pacientes foram operados para a correção da rotura miocárdica, sendo 5 $(20,8 \%)$ em sua forma aguda e $19(79,2 \%)$ na subaguda. Dentre os operados, $11(45,8 \%)$ tinham lesão em apenas uma artéria coronária, 3 (12,5\%) em duas artérias e $10(41,7 \%)$ em três artérias coronárias.

\section{Técnica Cirúrgica}

A via de acesso cirúrgico foi a toracotomia mediana através de esternotomia convencional em todos os pacientes. A circulação extracorpórea foi utilizada em $16(66,7 \%)$ dos pacientes, tendo sido estabelecida através de canulação da artéria e da veia femoral em $11(68,7 \%)$ pacientes, sendo 5 portadores de rotura aguda e 6 de rotura subaguda, que se apresentavam hemodinamicamente instáveis. Em $5(31,3 \%)$ pacientes portadores de rotura subaguda, com quadro clínico estável, a circulação extracorpórea foi estabelecida convencionalmente, através de canulação da aorta ascendente e do átrio direito. O local da rotura foi identificado visualmente e suas bordas palpadas a fim de se delimitar as margens do tecido necrótico e do miocárdio viável.

Em $3(18,7 \%)$, dentre os 16 pacientes operados com circulação extracorpórea, foi utilizada a técnica de pinçamento intermitente da aorta e, nos outros $13(81,3 \%)$, utilizou-se solução cardioplégica sangüínea hipotérmica para proteção miocárdica.

O reparo da rotura cardíaca, nesses 16 pacientes, foi realizado pela rafia da área acometida, através da aproximação das bordas rotas com fios inabsorvíveis de Polipropileno 2.0, ancorados em tiras de Teflon (Figuras 3 a 6). 
Abreu Filho C A C, Dallan L A O, Lisboa L A F, Platania F, Iglézias J C R, Cabral R H, Bordallo R, Dallan L A P, Oliveira S A Tratamento cirúrgico da rotura de parede livre do ventrículo esquerdo após infarto agudo do miocárdio. Rev Bras Cir Cardiovasc 2002; 17(1): 6-12.

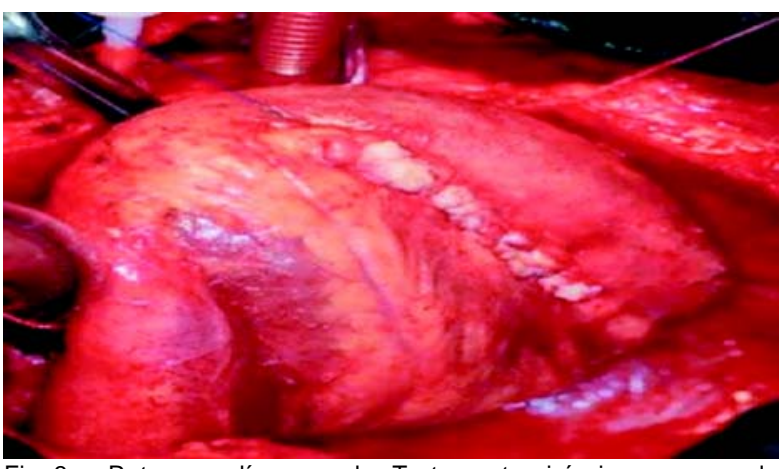

Fig. 3 - Rotura cardíaca aguda. Tratamento cirúrgico com uso de circulação extracorpórea.

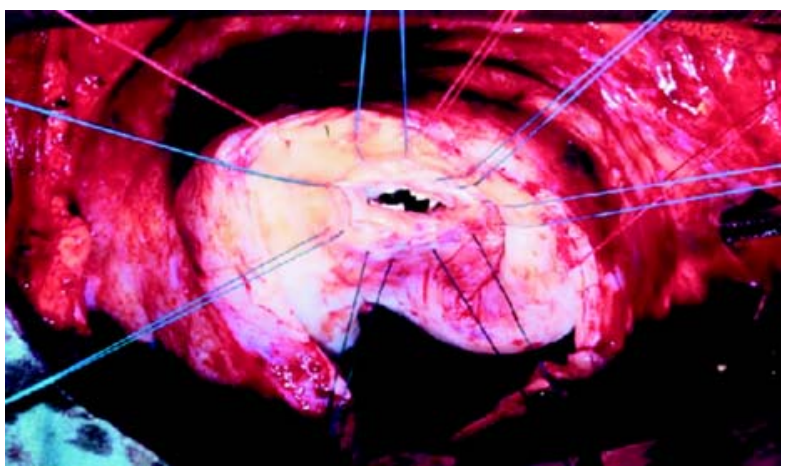

Fig. 4 - Rotura cardíaca subaguda. "Pseudo-aneurisma ventricular" tratamento cirúrgico.

Em $8(33,3 \%)$ pacientes, todos portadores de rotura cardíaca subaguda, não se utilizou a circulação extracorpórea. Nestes, de casuística mais recente, a infartectomia não foi realizada, e o reparo da área rota do ventrículo esquerdo foi procedido através da aplicação direta de cola de cianoacrilato sobre a área rota e nos tecidos infartados adjacentes, sendo recoberta com um retalho de pericárdio bovino conservado em glutaraldeído.

A análise estatística foi realizada através do método do Qui-quadrado e do teste exato de Fisher. Foram considerados significativos valores iguais ou inferiores a 0,05.

\section{RESULTADOS}

Observamos $98(77,2 \%)$ casos de rotura aguda da parede ventricular e $29(22,8 \%)$ casos em que ela foi subaguda. Pudemos constatar 97 (98,9\%) óbitos no grupo de pacientes com rotura aguda e 13 $(44,8 \%)$ óbitos dentre os pacientes com rotura cardíaca subaguda. No total, $110(86,6 \%)$ dos 127 pacientes estudados faleceram, com ampla prevalência dos portadores de rotura aguda.

A parede anterior do ventrículo esquerdo esteve comprometida em 59 (46,5\%) pacientes, a lateral em 52 (41\%) e a póstero-inferior em 16 (12,5\%) (Gráfico 1).

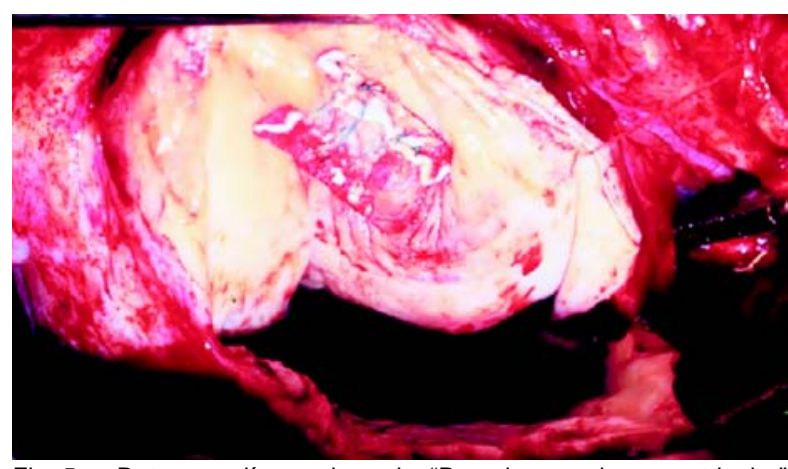

Fig. 5 - Rotura cardíaca subaguda. "Pseudo-aneurisma ventricular" tratamento cirúrgico.

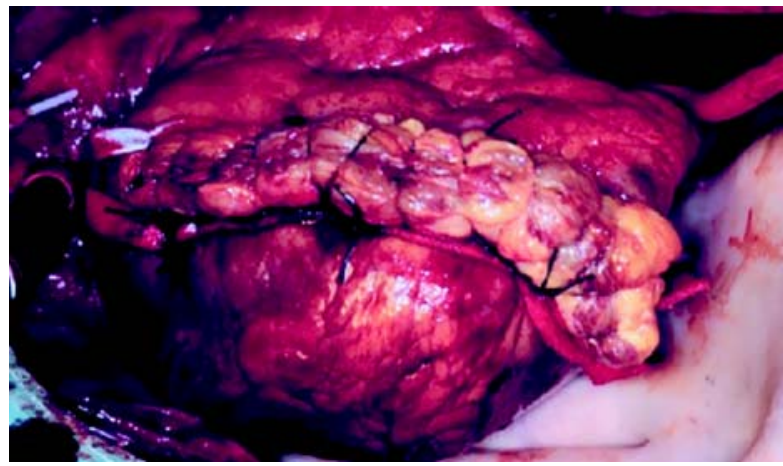

Fig. 6 - Rotura cardíaca subaguda. "Pseudo-aneurisma ventricular" tratamento cirúrgico concluído.

O tempo mínimo decorrido entre o IAM e a rotura ventricular foi de 2 horas e o máximo de 11 dias, com média de 2,37 dias.

Doença aterosclerótica de grau severo (obstrução de $70 \%$ ou mais da luz do vaso) comprometendo apenas uma artéria coronária foi observada em $43(33,8 \%)$ pacientes. Em 21 (16,5\%) pacientes havia comprometimento bi-arterial, nos 63 (49,7\%) restantes havia três ou mais artérias coronárias com lesões significativas.

Com relação ao uso da terapêutica trombolítica para tratamento do IAM, a rotura cardíaca ocorreu nos primeiros 5 dias após o IAM em 27 (96,4\%) dos 28 pacientes que receberam trombolíticos e, após o quinto dia, em apenas 1 (3,6\%) paciente. Dentre os 99 pacientes que não receberam terapêutica trombolítica, a rotura se deu nos primeiros 5 dias após o IAM em $61(61,6 \%)$ pacientes e foi tardia (após o quinto dia) nos $38(38,4 \%)$ restantes $(p=0,006)$ (Gráfico 2$)$.

Vinte e quatro $(18,9 \%)$ pacientes foram operados visando ao reparo da rotura miocárdica, dos quais $7(29,2 \%)$ faleceram. Desse total, $19(79,2 \%)$ constituíam-se no grupo de rotura subaguda, dos quais $3(15,8 \%)$ faleceram. Dentre os $5(20,8 \%)$ pacientes do grupo de rotura aguda operados, 4 $(80,0 \%)$ faleceram. O teste exato de Fisher revelou diferença significativa de mortalidade hospitalar entre os dois grupos ( $p=0,0016)$ (Gráfico 3 ). 
Abreu Filho C A C, Dallan L A O, Lisboa L A F, Platania F, Iglézias J C R, Cabral R H, Bordallo R, Dallan L A P, Oliveira S A Tratamento cirúrgico da rotura de parede livre do ventrículo esquerdo após infarto agudo do miocárdio. Rev Bras Cir Cardiovasc 2002; 17(1): 6-12.

\section{GRÁFICO 1}

DISTRIBUIÇÃO DA ROTURA CARDÍACA DE ACORDO COM A PAREDE DO VENTRÍCULO ESQUERDO ACOMETIDA

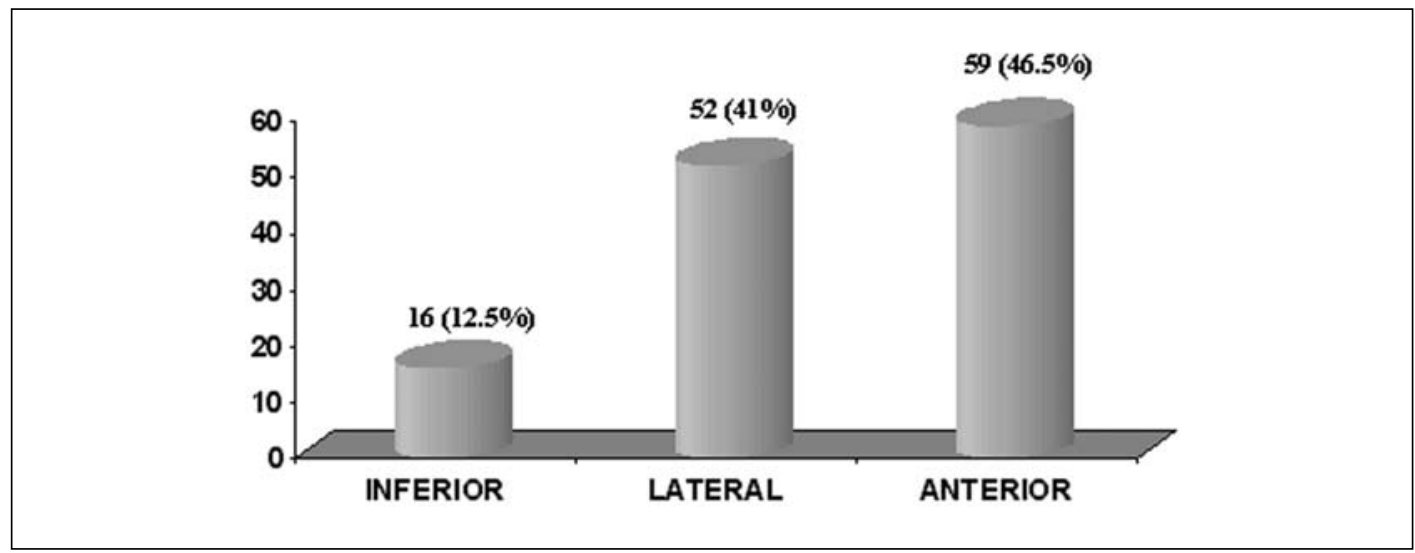

\section{GRÁFICO 2}

MOMENTO DA ROTURA CARDÍACA DE ACORDO COM O USO DE TERAPÊUTICA TROMBOLITICA

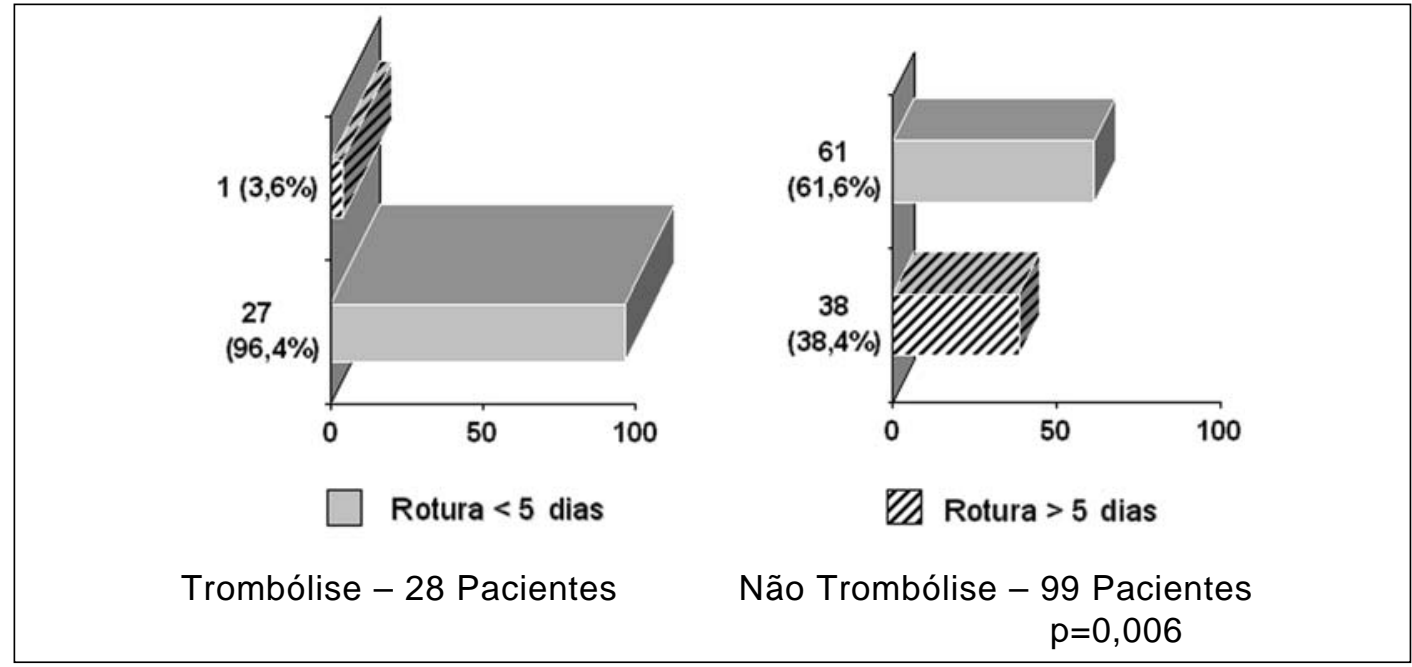

GRÁFICO 3

MORTALIDADE HOSPITALAR DE ACORDO COM O TIPO DE ROTURA CARDÍACA

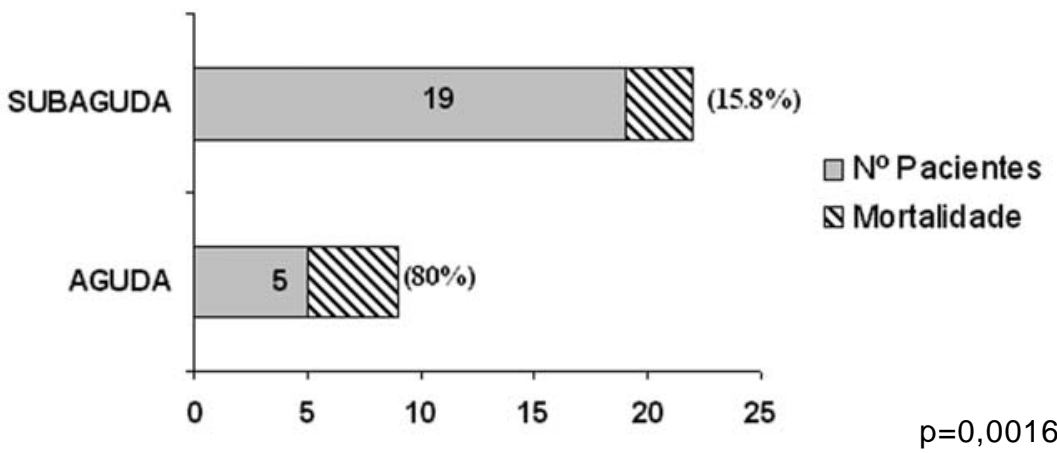


Abreu Filho C A C, Dallan L A O, Lisboa L A F, Platania F, Iglézias J C R, Cabral R H, Bordallo R, Dallan L A P, Oliveira S A Tratamento cirúrgico da rotura de parede livre do ventrículo esquerdo após infarto agudo do miocárdio. Rev Bras Cir Cardiovasc 2002; 17(1):6-12.

\section{COMENTÁRIOS}

A rotura de parede livre ventricular representa uma emergência cirúrgica de alta gravidade, especialmente quando do tipo aguda. Nessa situação, com freqüência, o diagnóstico não é estabelecido em tempo hábil para que se promova um procedimento cirúrgico de salvamento.

As manifestações clínicas iniciais da rotura cardíaca podem incluir sinais de tamponamento cardíaco, como abafamento de bulhas cardíacas, estase venosa jugular e pulso paradoxal (5). A estes achados se associa a dor torácica inespecífica e a hipotensão arterial sistêmica. O sinal clínico mais fidedigno de rotura de parede livre ventricular é a dissociação eletromecânica, que apresenta 97,6\% de acurácia diagnóstica (6).

Dentre os exames complementares, o mais importante para o diagnóstico da rotura cardíaca é o ecocardiograma, o qual pode ser realizado nas unidades de atendimento médico de emergência, possibilitando a detecção precoce dessa grave complicação do IAM. Isso tem contribuído para elevar os índices de tratamento cirúrgico imediato com sucesso. A expansão da parede miocárdica infartada associada à efusão pericárdica são achados ecocardiográficos típicos de rotura de parede livre ventricular. A visibilização direita da rotura da parede ventricular constitui sinal incontestável de sua presença (7-9).

A incidência da rotura de parede livre do ventrículo esquerdo oscila entre 1 a $4 \%$ dos pacientes atendidos com diagnóstico de IAM (10), o que está de acordo com nossa casuística, em que 127 (1,02\%) pacientes apresentaram rotura cardíaca, dentre os 12.405 pacientes atendidos no InCor com diagnóstico de IAM, entre janeiro de 1983 e maio de 1999.

A rotura cardíaca apresenta elevada mortalidade hospitalar, tendo superado as arritmias cardíacas, constituindo-se na segunda causa de morte em pacientes na fase aguda do infarto do miocárdio $(2,4)$. Trata-se da mais grave complicação mecânica do IAM, sendo 3 a 10 vezes mais freqüente do que a rotura do músculo papilar da valva mitral ou do septo interventricular (11).

A rotura cardíaca é mais freqüente em pacientes que sofreram o primeiro episódio de IAM, muitos deles sem manifestações clínicas pregressas ${ }^{(12,13)}$. Esse fato, pode ser explicado pela menor quantidade de circulação colateral no miocárdio, que não sofreu episódio isquêmico prévio(14). MANN \& ROBERTS (15), observaram que, dentre 138 pacientes portadores de rotura da parede livre do ventrículo esquerdo, apenas $13 \%$ apresentavam infartos antigos.

A influência da reperfusão miocárdica através de medicamentos trombolíticos na incidência de rotura cardíaca ainda é controversa. Existem indícios de que a rotura cardíaca ocorra mais precocemente nos casos em que se empregou a terapêutica trombolítica após o IAM (16). Ao analisarmos os nossos resultados, observamos que 27 (96,4\%), dentre os 28 pacientes que receberam terapêutica trombolítica, a rotura ocorreu nos primeiros 5 dias após o IAM. Nos demais 99 pacientes, que não receberam essa terapêutica, a rotura se deu nos primeiros 5 dias em apenas $61(61,6 \%)$ dos casos.

Com relação ao grau de comprometimento arterial coronário, os dados da literatura são controversos. Alguns autores afirmam que a rotura miocárdicas incide preferencialmente em pacientes uniarteriais (14). Outros sugerem a prevalência de doença aterosclerótica coronária triarterial (17). Em nossa casuística, $33,8 \%$ dos pacientes eram portadores de coronariopatia uniarterial.

A parede miocárdica anterior é apontada por diversos autores como a mais susceptível de sofrer rotura cardíaca (17); entretanto, autores como PADRÓ et al. ${ }^{(18)}$ advogam que a rotura cardíaca pode ocorrer igualmente em qualquer região do ventrículo esquerdo; outros autores observaram maior acometimento de rotura na parede ventricular lateral (44\%) (17). Esse índice é semelhante ao que observamos em nossa casuística (41\%). Provavelmente, o aumento do stress na parede lateral do miocárdio, resultante da contração dos dois músculos papilares adjacentes à valva mitral, facilitaria o desencadeamento da rotura.

Muitos pacientes com rotura da parede livre do miocárdio morrem subitamente, muitas vezes ainda sem diagnóstico. Em nossa casuística, observamos $98(77,2 \%)$ pacientes nessas condições, dos quais apenas $5(5,1 \%)$ foram operados, com 1(20\%) sobrevivente. Esses números demonstraram a gravidade da rotura cardíaca aguda.

A forma subaguda, por sua vez, tem instalação mais lenta, o que possibilita sua identificação e permite uma eventual correção cirúrgica. Sua incidência exata não está bem determinada; alguns autores estimam em $30 \%$ a ocorrência de rotura cardíaca subaguda após IAM(19). Pudemos observar que, dentre os nossos 127 pacientes, $29(22,8 \%)$ apresentavam rotura subaguda, sendo que $19(65,5 \%)$ foram submetidos ao tratamento cirúrgico, com sobrevida hospitalar de $84,2 \%$. Resultados satisfatórios também foram obtidos por outros autores no tratamento cirúrgico da rotura ventricular subaguda, com $76 \%$ de sobrevida hospitalar e $48,5 \%$ de sobrevida após seguimento médio de 30 meses $^{(19)}$.

O tratamento da rotura de parede livre ventricular é essencialmente cirúrgico. Nos casos de rotura aguda, que invariavelmente evoluem rapidamente para o choque cardiogênico e parada cardiorres- 
Abreu Filho C A C, Dallan L A O, Lisboa L A F, Platania F, Iglézias J C R, Cabral R H, Bordallo R, Dallan L A P, Oliveira S A Tratamento cirúrgico da rotura de parede livre do ventrículo esquerdo após infarto agudo do miocárdio. Rev Bras Cir Cardiovasc 2002; 17(1): 6-12.

piratória, o melhor recurso é se tentar obter suporte hemodinâmico através da instalação da circulação extracorpórea pela via fêmoro-femoral( ${ }^{(20)}$. Na nossa experiência, a circulação extracorpórea foi estabelecida por essa via em 5 pacientes portadores de rotura cardíaca aguda e em 6 pacientes portadores de rotura cardíaca subaguda com quadro clínico instável. Nos primeiros pacientes de nossa casuística, a correção cirúrgica se fez com auxílio da circulação extracorpórea e através da infartectomia da área acometida. Na década de 80 , foram propostas técnicas que buscavam o tamponamento da área rota, através da sutura de retalho de Teflon sobre o miocárdio normal subjacente ${ }^{(21)}$. Com isso, evitava-se a infartectomia local.

Com o desenvolvimento de colas biológicas, procedimentos alternativos ao uso da circulação extracorpórea e que evitavam a infartectomia passaram a ser utilizados(18). O emprego da cola biológica de cianoacrilato associado à colocação de um retalho de pericárdio bovino sobre a área rota e nos tecidos infartados adjacentes substituiu a sutura de Polipropileno sobre o miocárdio friável, evitando os sangramentos decorrentes do seu esgarçamento. Esse método tem-se mostrado o mais eficiente para controlar o sangramento e prevenir a recorrência da rotura (20).

PADRÓ et al. (18) relataram o tratamento de 13 pacientes portadores de rotura subaguda de ventrículo esquerdo utilizando essa técnica, com $100 \%$ de sobrevida a longo prazo.

Podemos concluir que a rotura de parede livre do ventrículo esquerdo é uma grave complicação do IAM, sendo o ecocardiograma o principal e mais disponível método diagnóstico. As formas agudas estão associadas a alta mortalidade, dada a rapidez de sua evolução e a dificuldade de sua correção cirúrgica. A rotura subaguda tem melhor prognóstico, especialmente se detectada precocemente. Sua correção, através de métodos que dispensam a circulação extracorpórea, tem apresentado resultados cirúrgicos satisfatórios.

RBCCV 44205-566

Abreu Filho C A C, Dallan L A O, Lisboa L A F, Platania F, Iglézias J C R, Cabral R H, Bordallo $R$, Dallan $L A P$, Oliveira $S A$ - Surgical treatment of left ventricular free wall rupture after acute myocardial infarction. Rev Bras Cir Cardiovasc 2002; 17(1): 6-12.

ABSTRACT:Material and Methods: Between January 1983 and May 1999, 12.405 patients were treated by the surgical team of the Heart Institute (InCor) with the diagnosis of acute myocardial infarction (AMI). From these patients, $127(1.02 \%)$ had left ventricular free wall rupture as an ischemic complication of the myocardial infarction. The cardiac rupture was acute in 98 patients $(77.1 \%)$ and sub-acute in $29(22.9 \%)$.

Results: Twenty-four patients were operated on, 5 on acute rupture with $80 \%$ of hospital mortality and 19 on sub-acute rupture with $15.8 \%$ of hospital mortality. The post-operative overall survival including both groups was $70.8 \%$.

Conclusion: The conclusion was drawn that left ventricular free wall rupture is a severe complication of acute myocardial infarction that needs an immediate action. In acute ruptures, most patients develop hemodynamic deterioration without enough time to try to proceed any surgical correction. The sub-acute cases can be detected and monitored through periodic ecocardiographic exams after the AMI. In these cases the early surgical intervention, many times without using extra-corporeal circulation, has been increasing the chances of survival of the majority of these patients.

DESCRIPTORS: Heart ventricle, surgery. Myocardial infarction, complications. Heart rupture, post-infartion. 
Abreu Filho C A C, Dallan L A O, Lisboa L A F, Platania F, Iglézias J C R, Cabral R H, Bordallo R, Dallan L A P, Oliveira S A Tratamento cirúrgico da rotura de parede livre do ventrículo esquerdo após infarto agudo do miocárdio. Rev Bras Cir Cardiovasc 2002; 17(1): 6-12.

\section{REFERÊNCIAS BIBLIOGRÁFICAS}

1 Harvey W - De Circulatio Sanguinis. Exercit 3. Citado por Morgagni GB In: The seats and causes of diseases. Tradução Benjamin Alexander. London: Letter 27, 1769: 830.

2 Reardon M J, Carr C L, Diamond A et al. - Ischaemic left ventricular free wall rupture: prediction, diagnosis and treatment. Ann Thorac Surg 1997; 64: 1509-13.

3 Reddy S G \& Roberts W C - Frequency of rupture of the left ventricular free wall or ventricular septum among necropsy cases of fatal acute myocardial infarction since introduction of coronary care units. Am J Cardiol 1989; 63: 906-11.

4 Nakamura F.; Minamino T.; Higashino $Y$ et al. - Cardiac free wall rupture in acute myocardial infarction: ameliorative effect of coronary reperfusion. Clin Cardiol 1992; 15: 244-50.

5 Sutherland F W, Guell F J, Pathi V L, Naik S K - Postinfarction ventricular free wall rupture: strategies for diagnosis and treatment. Ann Thorac Surg 1996; 61: 1281-5.

6 Figueras J, Curos A, Cortadellas J, Sans M, Soler-Soler $\mathrm{J}$-Relevance of electrocardiographic findings, heart failure and infarct site in assessing risk and timing of left ventricular free wall rupture during acute myocardial infarction. Am J Cardiol 1995; 76: 543-7.

7 Desoutter $\mathrm{P}$, Halphen $\mathrm{C}$, Haiat $\mathrm{R}$ - Two dimension echocardiographic visualization of free ventricular wall rupture in acute anterior myocardial infarction. $A m$ Heart J 1984; 108: 1360-1.

8 Chirillo F, Cavarzerani A, Lus P et al. - Role of transthoracic, transesophageal and transgastric twodimensional and color doppler echocardiography in the evaluation of mechanical complications of acute myocardial Infarction. Am J Cardiol 1995; 76: 833-6.

9 Shapira I, Isakov A, Burke A C - Cardiac rupture in patients with acute myocardial infarction. Chest 1987; 92: 219-23.

10 Rasmussen S, Leth A, Kjoller E, Pedersen A - Cardiac rupture in myocarcial infarction: a review of 72 consecutive cases. Acta Med Scand 1979; 205: 11-6.

11 Dallan L A, Oliveira S A, Ramires J A F et al. - Tratamento cirúrgico da comunicação interventricular pós infarto agudo do miocárdio. Rev Bras Cir Cardiovasc 1989; 4: 64-74.
12 Ishibashi-Ueda H, Imakita M, Fujita H, Katsuragi M, Yutani C - Cardiac rupture complicating hemorrhagic infarction after intracoronary thrombolysis. Acta Pathol Jpn 1992; 42: 504-7.

13 Figueras J, Cortadellas J, Calvo F, Soler-Soler J Relevance of delayed hospital admission on development of cardiac rupture during acute myocardial infarction: study in 225 patients with free wall, septal or papillary muscle rupture. J Am Coll Cardiol 1998; 32: 135-9.

14 Schwarz C D, Punzengruber C, Ng C K, Schauer N, Hartl $\mathrm{P}$, Pachinger $\mathrm{O}-$ Clinical presentation of rupture of the left ventricular free wall after myocardial infarction: report of five cases with successful surgical repair. Thorac Cardiovasc Surg 1996; 44: 71-5.

15 Mann J M \& Roberts W C - Rupture of the left ventricular free wall during acute myocardial infarction: analysis of 138 necropsy patients and comparison with 50 necropsy patients with acute myocardial infarction without rupture. Am J Cardiol 1988; 62: 847-59.

16 Becker R C, Charlesworth A, Wilcox R G et al. - Cardiac rupture associated with thrombolitic therapy: impact of time to treatment in the late assessment of thrombolitic efficacy (LATE) study. J Am Coll Cardiol 1995; 25: 1063-8.

17 Batts K, Ackermann D M, Edwards W D - Postinfarciton rupture of the left ventricular free wall: clinicopathologic correlates in 100 consecutive autopsy cases. Hum Pathol 1990; 21: 530-5.

18 Padró J M, Mesa J M, Silvestre J et al. - Subacute cardiac rupture: repair with a sutureless technique. Ann Thorac Surg 1993; 55: 20-4.

19 López-Sendón J, Gonçalvez A, Lopez de Sá E et al. - Diagnosis of subacute ventricular wall rupture after acute myocardial infarction sensitivity and specificity of clinical, hemodynamic and echocardiographic criteria. J Am Coll Cardiol 1992; 19: 1145-53.

20 McMullan M H, Maples M D, Kilgore T L Jr, Hindman S H - Surgical experience with left ventricular free wall rupture. Ann Thorac Surg 2001; 71:1894-9.

21 Nunez L, de la Llana R, López-Sendón J, Coma I, Gil Aguado M, Larrea J L - Diagnosis and treatment of subacute free wall ventricular rupture after infarction. Ann Thorac Surg 1983; 35: 525-9. 